\title{
Sleep-dependent Neurophysiological Processes in Implicit Sequence Learning
}

\author{
Charline Urbain ${ }^{1,2}$, Rémy Schmitz ${ }^{1}$, Christina Schmidt ${ }^{3}$, \\ Axel Cleeremans ${ }^{4}$, Patrick Van Bogaert ${ }^{2}$, Pierre Maquet ${ }^{3}$, \\ and Philippe Peigneux ${ }^{1,3}$
}

\begin{abstract}
Behavioral studies have cast doubts about the role that posttraining sleep may play in the consolidation of implicit sequence learning. Here, we used event-related fMRI to test the hypothesis that sleep-dependent functional reorganization would take place in the underlying neural circuits even in the possible absence of obvious behavioral changes. Twenty-four healthy human adults were scanned at Day 1 and then at Day 4 during an implicit probabilistic serial RT task. They either slept normally (RS) or were sleep-deprived (SD) on the first posttraining night. Unknown to them, the sequential structure of the material was based on a probabilistic finite-state grammar, with $15 \%$ chance on each trial of replacing the rules-based grammatical $(G)$ stimulus with a nongrammatical (NG) one. Results indicated a gradual differentiation across sessions
\end{abstract}

\section{INTRODUCTION}

Memory consolidation can be defined as the gradual offline process whereby, in the absence of further practice, novel memory traces become more stable and resistant to interference with the passage of time (McGaugh, 2000). In this respect, motor sequence learning not only takes place during practice but also continues to be consolidated in memory during postlearning periods. However, how and whether offline periods of sleep and wakefulness respectively contribute to the consolidation of motor sequence learning remain a matter of debate. Whereas several studies have found that postlearning sleep contributes to the consolidation or stabilization of elementary motor skills in young adults (Barakat et al., 2011; Debas et al., 2010; Fischer, Hallschmid, Elsner, \& Born,

\footnotetext{
${ }^{1}$ Neuropsychology and Functional Neuroimaging Research Unit at CRCN - Center for Research in Cognition and Neurosciences and UNI - ULB Neurosciences Institute, Université Libre de Bruxelles (ULB), Brussels, Belgium ${ }^{2}$ Laboratoire de Cartographie Fonctionnelle du Cerveau, Hôpital Erasme, and UNI, ULB, Brussels, Belgium, ${ }^{3}$ Cyclotron Research Center, University of Liége, Liége, Belgium, ${ }^{4}$ Consciousness, Cognition \& Computation Group, at CRCN and UNI, ULB, Brussels, Belgium
}

between RTs (faster RTs for G than NG), together with NG-related BOLD responses reflecting sequence learning. Similar behavioral patterns were observed in RS and SD participants at Day 4, indicating time- but not sleep-dependent consolidation of performance. Notwithstanding, we observed at Day 4 in the RS group a diminished differentiation between G- and NG-related neurophysiological responses in a set of cortical and subcortical areas previously identified as being part of the network involved in implicit sequence learning and its offline processing during sleep, indicating a sleep-dependent processing of both regular and deviant stimuli. Our results suggest the sleep-dependent development of distinct neurophysiological processes subtending consolidation of implicit motor sequence learning, even in the absence of overt behavioral differences.
2002; Smith \& MacNeill, 1994), results are more controversial in the domain of visuomotor sequence learning. For instance, sleep-dependent consolidation and offline performance improvement were found for context-related (Spencer, Gouw, \& Ivry, 2007; Spencer, Sunm, \& Ivry, 2006) and goal-related (Cohen \& Robertson, 2007; Cohen, Pascual-Leone, Daniel, \& Robertson, 2005) components of an incidentally learned sequence. Contrarily, however, consolidation of the movement-related component for the same sequential material was found to develop over wakefulness only (Cohen \& Robertson, 2007; Cohen et al., 2005). Also, postlearning sleep contributes developing awareness about a sequence practiced during an implicit learning session (Fischer, Drosopoulos, Tsen, \& Born, 2006), and visuomotor sequence learning was found sleep-dependent only when learning was explicit (Robertson, Pascual-Leone, \& Press, 2004). These results led authors to propose that the mechanisms at work during postlearning sleep and wakefulness periods differentially impact the brain networks subtending the development of particular components of explicit and implicit motor sequence learning (Robertson, 2009). Discrepant results were also found using probabilistic versions of the serial RT task (SRTT), arguably the most implicit form of sequence learning task as participants 
consistently fail to exhibit signs of conscious knowledge of the underlying sequential regularities, despite a progressive differentiation between RTs for items following versus violating these sequential rules (Jimenez, Mendez, \& Cleeremans, 1996). Using probabilistic SRTT, performance improvement or stabilization was found after sleep (Fischer, Wilhelm, \& Born, 2007; Cajochen et al., 2003), but also after wakefulness and sleep (Nemeth et al., 2010), suggesting that both states may contribute to the consolidation of implicit motor sequence learning. Furthermore, Song, Howard, and Howard (2007) claimed that implicit probabilistic sequence learning does not benefit at all from sleep, with daytime enhancement occurring only for general skills but not for sequencespecific learning in probabilistic SRTT. Finally, it was suggested that both conscious awareness and attention are necessary components for the development of sleepdependent memory consolidation effects (Song, 2009; Robertson et al., 2004).

Noticeably, the conclusion that sleep does not participate in implicit sequence learning is partially discrepant with neuroimaging data yielding evidence for the reactivation of learning-related neuronal ensembles during rapid eye movement (REM) sleep after extended practice on this same probabilistic SRTT (Peigneux et al., 2003; Maquet et al., 2000). These studies also demonstrated that the reactivations are not merely related to the acquisition of basic visuomotor skills through practice but are contingent on the implicit acquisition of the probabilistic sequential regularities embedded in the material (Peigneux et al., 2003). Also, learning the SRTT eventually increased functional connectivity patterns in motor skillrelated networks during posttraining REM sleep (Peigneux et al., 2003; Laureys et al., 2001), and learning performance correlated with regional CBF in reactivated areas (Peigneux et al., 2003). Additionally, it was shown in a constant routine protocol that improvement in probabilistic sequence learning starts developing only after the occurrence of REM sleep in nap episodes (Cajochen et al., 2003).

Considering these divergent results, it can be argued that both sleep- and wake-dependent plasticity mechanisms participate in the consolidation of implicit motor sequence learning, which does not necessarily entail that qualitative contributions are the same. Hence, postlearning sleep might also exert a specific influence on the cerebral reorganization processes subtending the consolidation of implicitly learned sequential regularities. In this respect, it is conceivable to observe seemingly similar behavioral patterns after sleep than wakefulness postlearning episodes, but these behavioral patterns would be subtended by partially different brain activity profiles, a dissociation already demonstrated in the declarative memory domain (Rauchs et al., 2008; Gais et al., 2007; Orban et al., 2006). In this study, we tested the hypothesis that long-term sleep-dependent functional reorganization takes place in the neural circuits underlying implicit sequence learning even in the possible absence of detectable behavioral changes, using event-related fMRI in participants trained to the SRTT, then allowed to sleep or deprived of sleep on the following night, and then tested again 3 days later.

\section{METHODS}

Twenty-four right-handed (laterality score: mean $=80.8$, $S D=18.6$; Oldfield, 1971) healthy volunteers (five men, mean age $=21.7$ years, range $=19-25$ years) gave their informed consent to participate in this fMRI experiment approved by the ethical committee of the University of Liege. None of them reported any neurological or psychiatric disease history nor used any centrally acting medication for the time of the experiment. All participants were asked to be stimulant drug-free (e.g., caffeine) before each testing session. No participant was trained as a professional typist or musician.

Circadian chronotype and sleep quality over the month preceding the experiment were assessed using a Morningness-Eveningness Self-assessment (Horne \& Ostberg, 1976) and the Pittsburgh Sleep Quality Index (Buysse, Reynolds, Monk, Berman, \& Kupfer, 1989) questionnaires, respectively. Sleep quality and quantity for the nights preceding each of the four experimental days (i.e., the night preceding [N-1] learning and the three subsequent nights [N1 with or without sleep deprivation and N2-N3 with regular sleep]) were monitored using the St Mary's Hospital sleep questionnaire (Ellis et al., 1981). Participants were also instructed not to take a nap during this period. Compliance to a regular sleepwake schedule was monitored using wrist actimetry (Daqtix, Oetzen, Germany) for the four days preceding the first learning session until the end of the experiment.

\section{Probabilistic SRTT}

Implicit sequence learning was assessed through the probabilistic version of the SRTT (Peigneux et al., 2003; Jimenez et al., 1996) used in previous PET studies showing experience-dependent reactivations during sleep (Peigneux et al., 2003; Maquet et al., 2000). The implicit nature of the knowledge about the sequential regularities gained in the probabilistic SRTT was previously demonstrated. Using the same task, Peigneux et al. found that participants' performance in an explicit sequence generation task (see Peigneux et al., 2000, for a detailed description) was not above-random level even after extended practice (i.e., 14,960 trials, but only 4,680 trials were administered in this study), indicating that sequence knowledge gained during practice in the probabilistic SRTT is essentially implicit (Peigneux et al., 2000, 2003). Similarly, other studies (e.g., Jimenez et al., 1996; Cleeremans \& McClelland, 1991) have consistently established the 
implicit nature of the relationships gained using this version of the probabilistic SRTT.

In the SRTT, participants were presented six permanent position markers, horizontally displayed on the fMRI scanner-compatible screen. The three left (respectively, right) position markers corresponded to three spatially compatible response keys using the left (respectively, right) index, middle, and ring fingers. A single SRT block consisted of 65 successive trials. On each trial, a black dot appeared $2 \mathrm{~cm}$ below one of the position markers. The task consisted of pressing as fast and accurately as possible on the corresponding key. Afterward, the next stimulus was displayed following a 200-msec response stimulus interval. Before fMRI sessions and SRTT practice, participants were told that the goal of the experiment was to study the brain correlates of sustained practice on a simple visuomotor task.

Unknown to them, however, the sequential structure of the material was based on a finite-state grammar that defined legal transitions between successive trials (Figure 1). To assess learning of the conditional probabilities for stimuli succession, there was a $15 \%$ chance on each trial of replacing the rules-based grammatical $(G)$ stimulus by a nongrammatical (NG), randomly selected stimulus. Assuming that (implicit) response preparation is facilitated by high predictability, G stimuli should thus elicit faster responses than NG stimuli, but only if the context in which the stimuli may occur has been encoded by participants. In this task, contextual sensitivity emerges through practice as a gradually increasing difference between the RTs elicited by $G$ and NG stimuli occurring in specific contexts set by the preceding trials (Jimenez et al., 1996; Cleeremans \& McClelland, 1991). Hereafter, we estimated, in each block, the differences between RTs elicited by $G$ and NG stimuli in the temporal context defined by the previous stimulus. The mapping between grammar elements and screen locations was systematically counterbalanced across participants.

\section{Experimental Procedure}

All volunteers were scanned using fMRI on Day 1 (Sessions [S]1 and S2) and Day 4 (S3) while performing the SRTT. Each fMRI session consisted of 24 blocks of 65 trials. Two successive learning sessions were administered at Day 1. S1 mostly captured the early stages of learning with initial adaptation processes to the task and development of basic visuo-motor adaptation skills. S2 accounted for a more stabilized pattern in which dissociation between $G$ and NG items in the sequence and high-order sequence learning abilities are better expressed. After completion of each block, the computer displayed information about
Figure 1. Sequence learning task and experimental protocol. (A) Probabilistic SRTT. Participants are instructed to press as fast and accurately as possible the key spatially corresponding to the location of the red dot on the computer screen (letters are shown for indicative purpose but actually not displayed to the participant), and then, the next stimulus is presented. Unknown to participants, the succession of screen locations follows a probabilistic sequential pattern based on the illustrated finite-state grammar (Jimenez et al., 1996). To assess learning of the conditional probabilities regulating stimuli succession, there is a $15 \%$ chance in each trial to replace the rules-based G stimulus by an NG, randomly selected location, allowing comparison of response speed and accuracy. (B) Experimental design. At Day 1, participants practiced two SRT sessions (S1, S2) consisting of 24 blocks each (65 trials/block) in the fMRI environment. At Day 4 and at the same time of day than Day 1, participants practiced an additional 24-block session (S3). During the first posttraining night, 12 participants were allowed to sleep regularly (RS), whereas the remaining 12 were SD under controlled conditions. All participants slept then normally for the two following nights before S3.

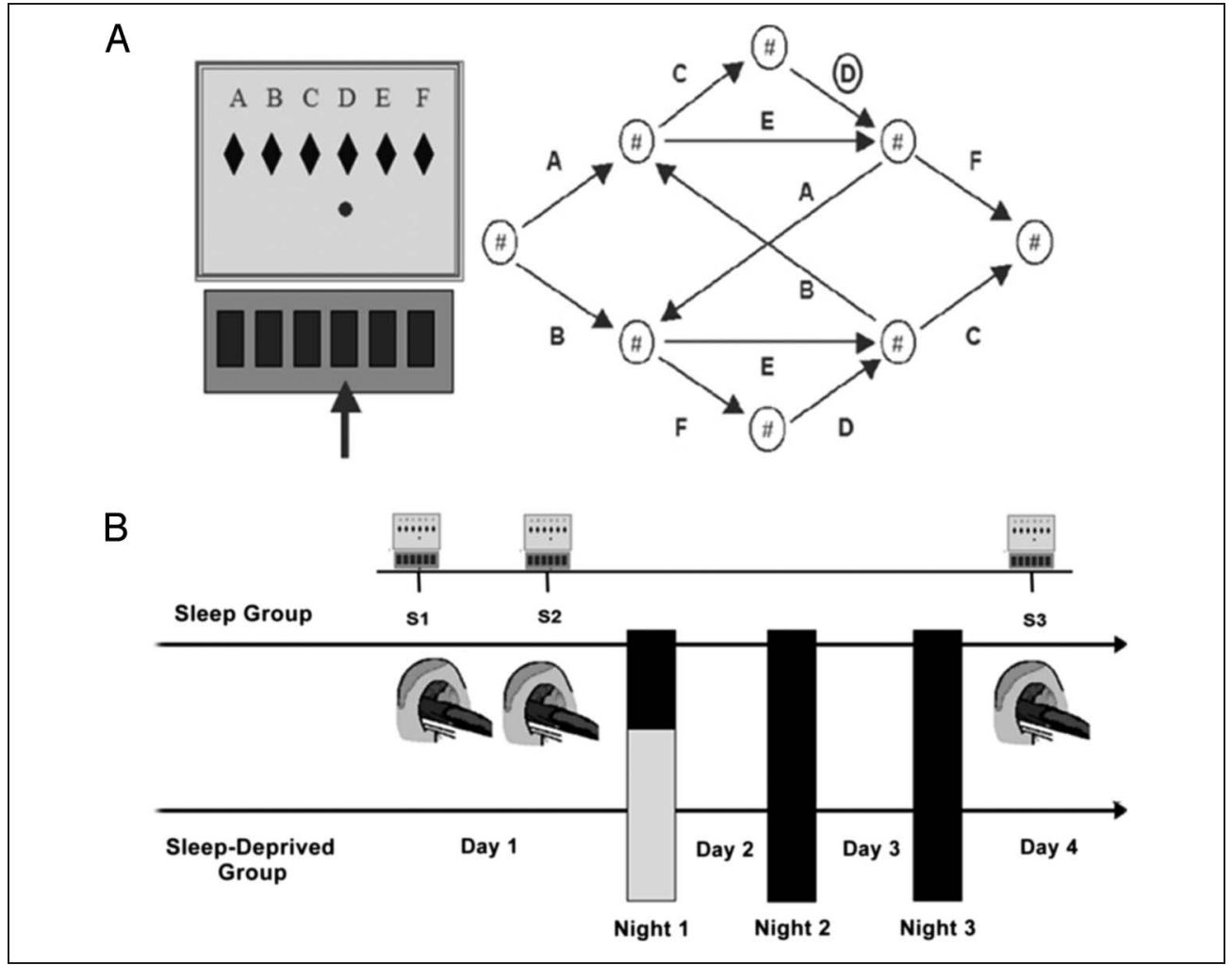


the participant's mean RT and accuracy as well as the evolution of performance (same, better, or worse) as compared with the preceding block. This summary feedback was given to encourage participants to continue performing both fast and accurately on the SRTT. Between-block intervals had a random duration between 8 and $13 \mathrm{sec}$, and a fixation cross replaced feedback information 2 sec before starting the new block.

After the completion of S1-S2 at Day 1, half of the participants were randomly assigned to the regular sleep (RS) or to the sleep-deprived (SD) condition. In the RS condition, participants were allowed to sleep as usual at home for three nights after training. In the SD condition, participants were kept awake in the laboratory during the first posttraining night under controlled conditions (low luminance, hourly isocaloric snacks, free water but no stimulant drinks, and only quiet activities allowed, e.g., reading or watching movies) and then had two recovery nights at home. All participants were then retested on Day 4 (S3) while performing the SRTT in the fMRI environment (Figure 1). To control for potential circadian interferences, all sessions for one participant took place at the same time of the day (between 10:00 a.m. and 5:00 p.m.) at Day 1 (S1-S2) and Day 4 (S3).

\section{Functional Imaging Parameters}

fMRI series were acquired using a head-only 3-T scanner (Siemens Allegra, Erlangen, Germany). Multislice T2*weighted fMRI images were obtained with a gradient-echo planar sequence using axial slice orientation (repetition time $=2130 \mathrm{msec}$, echo time $=40 \mathrm{msec}$, flip angle $=$ $90^{\circ}, 32$ transverse slices, 3-mm slice thickness, 30\% interslice gap, field of view $=220 \times 220 \mathrm{~mm}^{2}$, matrix size $=$ $64 \times 64 \times 32$, voxel size $=3.4 \times 3.4 \times 3.0 \mathrm{~mm}^{3}$ ). The four initial scans were discarded to avoid T1 saturation effects. For anatomical reference, a 3-D T1-weighted image was acquired for each participant (repetition time $=$ $1.96 \mathrm{msec}$, echo time $=4.43 \mathrm{msec}$, flip angle $=8^{\circ}$, field of view $=230 \times 173 \mathrm{~mm}^{2}$, matrix size $=256 \times 192 \times$ 176 , voxel size $=0.9 \times 0.9 \times 0.9 \mathrm{~mm}^{3}$ ).

\section{fMRI Data Analyses}

fMRI data were analyzed using SPM8 (www.fil.ion.ucl.ac. uk/spm) running on MATLAB 9 (Mathworks, Sherborn, MA). Preprocessing included realignment and adjustment for movement-related effects, coregistration of functional and anatomical data, normalization into standard stereotactic Montreal Neurological Institute (MNI) space, and spatial smoothing using a Gaussian kernel of 8-mm FWHM. Functional data were analyzed using a mixed-effect model aimed at showing stereotypical effect in the population from which the participants are drawn (Penny \& Holmes, 2003).

Because of the intrinsic properties of the probabilistic SRTT in which predictable G stimulus (85\%) are replaced by an NG stimulus in $15 \%$ of the case, the fast stimulus presentation rate at about 1 per second, and the time needed for whole-brain volume acquisition $(2.13 \mathrm{sec})$, only more sparsely distributed event-related responses for the $15 \%$ NG stimuli (indirectly reflecting learning of the sequential regularities) were modeled. Therefore, we investigated, for each individual and at each session (S1, S2, and S3), changes in BOLD responses in NG itemrelated cerebral regions on top of the motor practice including activity for $\mathrm{G}$ items within a block. This allowed us to identify cerebral regions in which BOLD response was modulated by grammatical violations in the sequence, using a canonical hemodynamic response function (HRF) and its onset latency (o) and temporal dispersion (d) derivatives (Friston et al., 1998). Additionally, practice blocks (including 85\% G items) were modeled using boxcar functions, and mean RTs per block were entered as confounding variables to control for unspecific motor speedrelated activations.

At the intraindividual (fixed-effect) level, brain responses for each participant were modeled at each voxel using a general linear model. NG items were modeled as a stick function representing its onset, and practice blocks were modeled using boxcar functions. The ensuing vectors were convolved with the canonical HRF and its temporal derivatives (onset latency [o] and dispersion [d]) and used as regressors in the individual design matrix. The incorporation of the time derivate term in the canonical HRF allows the peak response to vary by plus or minus a second, and incorporation of the dispersion derivative allows the width of the response to vary, thus allowing for limited variations in subject-by-subject and voxel-byvoxel responses (Friston et al., 1998). At each block, RTs were entered as parametric variates to control for motor speed-related activations. Movement parameters estimated during realignment (translations in $x, y$, and $z$ directions and rotations around $x, y$, and $z$ axes) were also included in the design matrix as a variable of no interest. Linear contrasts estimated the main effect of grammaticality deviations (NG) at S1, S2, and S3. High-pass filter was implemented using a cutoff period of 128 sec to remove low-frequency drifts from the time series. Serial autocorrelations were estimated with a restricted maximum likelihood algorithm using an autoregressive model of order 1 (white noise). The resulting set of voxel values constituted a map of $t$ statistics $[\operatorname{SPM}(t)]$ for each individual and parameter. Resulting individual contrast images (HRF, time, and dispersion derivatives separately computed for the NG contrast at S1, S2, and S3) were then spatially smoothed using a Gaussian kernel of 6-mm FWHM and entered in a between-individual second-level, random effects analysis (Penny \& Holmes, 2003). Because latency and dispersion derivative effects cannot be computed separately from their canonical HRF at the random effect level (Henson, Price, Rugg, Turner, \& Friston, 2002), we computed $F$ tests aimed at testing jointly relevant contrasts of parameter estimates. Contrast images 
were modeled at the random effect level using a factorial design with within-subject session (prenight [S2] vs. postnight [S3]) and responses (HRF, time, and dispersion derivatives) factors and between-subject posttraining sleep (RS vs. SD) factor. This interaction was masked exclusively ( $p<.001$, uncorrected) by initial between-group differences between SD and RS participants at the presleep session S2. Additionally, S1 was not included in this analysis as the early stages of SRTT practice might mostly account for basic visuo-motor adaptation skills, whereas high-order sequence learning components developed at a later stage are captured at S2 and S3. Inferences were drawn using $\operatorname{SPM}(F)$ tests accounting for effects combining HRF and its derivatives. Reported results are significant at the voxel level either at $p_{\text {corr }}<.05$ after correction for multiple comparisons in the whole-brain space or at $p_{\mathrm{svc}}<.05$ after correction in a small VOI (radius $=10 \mathrm{~mm}$ ) drawn around coordinates of interest taken from the literature. Results significant at the voxel level of $p<.001$ (uncorrected) are reported for the sake of completeness but not further discussed. Inspection of derivative parameter estimates allowed determining the direction of effects for NG-related BOLD responses, with positive parameters indicating earlier onset and smaller dispersion and, conversely, negative derivative BOLD responses indicating later onset and greater dispersion.

Additionally, a psychophysiological interaction (PPI) analysis (Friston et al., 1997) was computed to test the hypothesis that posttraining sleep would modulate functional connectivity between NG-related areas at S3. On the basis of the seed voxel statistically significant in the main random effects analysis after correction for multiple comparisons in the whole-brain volume $\left(p_{\text {corr }}<.05\right.$; see below), we computed the coordinates of the peak value for each participant inside a 6-mm spherical volume around the reference coordinate (provided that the identified location felt in the structure of interest, based on visual inspection of the participant's structural T1-weighted MRI). Individual time series for each location were obtained separately by extracting the first principal component from all raw voxel time series in a sphere (radius = $4 \mathrm{~mm}$ ) centered on the coordinate of the participantspecific peak value. These time series were meancorrected and high-pass filtered to remove low-frequency signal drifts. Then, the HRF was deconvolved from the BOLD time series to keep in the underlying neuronal time series. A new linear model was then built for each participant that incorporated the condition regressor (NG), the neuronal-activity regressor from the source area, and the psychophysiological regressor (i.e., the product from the interaction between the first psychological and the second physiological regressor) and the covariates of no interest (movement parameters) as in the initial model. After smoothing (6-mm FWHM Gaussian kernel), the individual summary statistic images of PPIs were entered into a second-level analysis, as described above, to evaluate group differences. For each between-group difference of brain activity coupling between a seed area and a functionally connected remote brain region, a plot of the mean size of effect within the two experimental populations was taken using SPM8 toolbox rfxplot (Gläscher, 2009) as an indicative measure of the group average direction (i.e., positive or negative) of coupling. Results were deemed significant at $p<.005$, uncorrected.

\section{RESULTS}

All participants had intermediate or neutral chronotype (Morningness-Eveningness Questionnaire score $=53.5$ [range $=35-65]$; Horne \& Ostberg, 1976). They kept a regular sleep schedule during the week preceding and throughout the experiment as measured by wrist actimetry and self-reports (average sleep duration $=7.4$ [range $=$ 7-9] hr, latency $=15.1$ [range $=3-45] \mathrm{min}$ ).

\section{Probabilistic SRT Performance}

Mean RTs for $G$ and NG stimuli were computed for each of the 24 SRT blocks ( 65 trials/block) practiced at

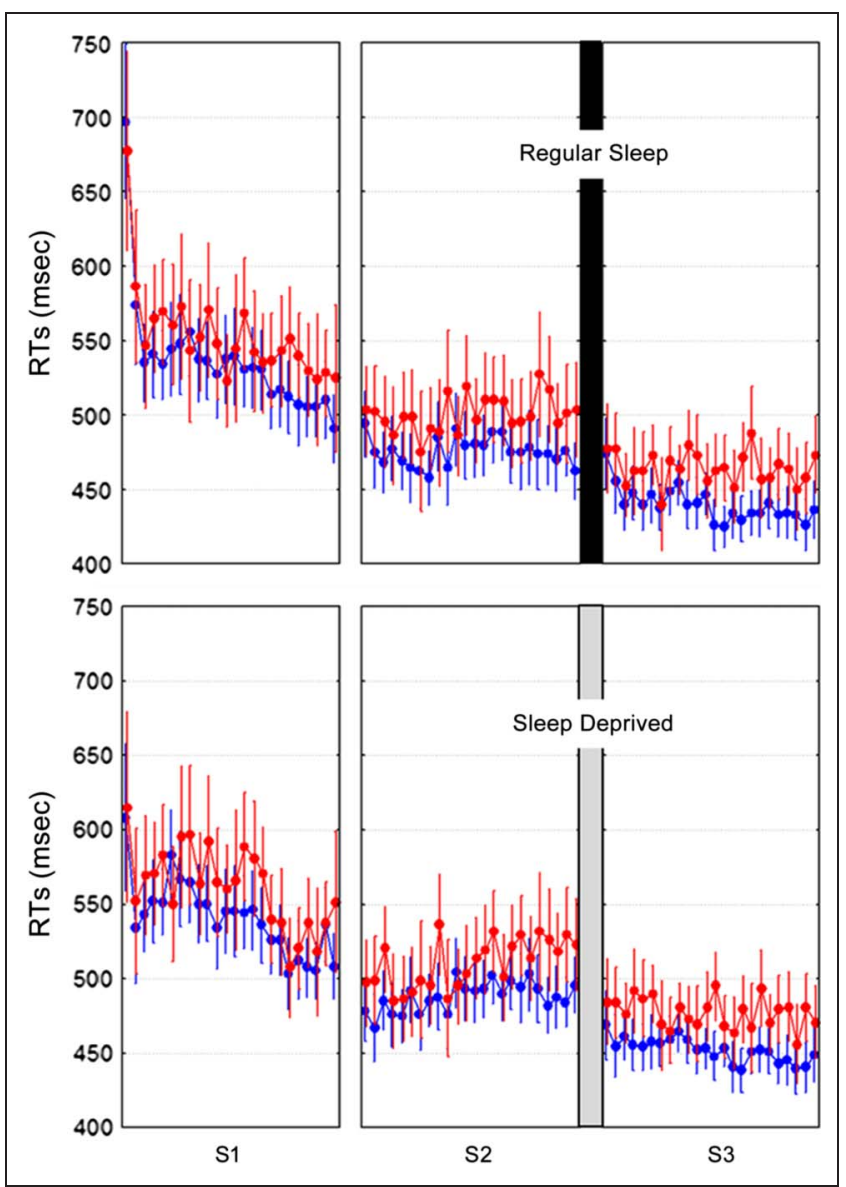

Figure 2. Behavioral results. Mean RTs (and standard errors of the mean) per block for $\mathrm{G}$ (in blue) and NG (in red) stimuli at learning $(\mathrm{S} 1, \mathrm{~S} 2)$ and retest (S3) sessions in RS and SD populations. 
Figure 3. (A) Grammatical violations-related brain network. (A, left) Statistical parametric map $\left(p_{\text {corr }}<.05\right)$ of grammatical violations-related BOLD responses across $\mathrm{S} 1$ and $\mathrm{S} 2$ at Day 1 during SRT practice, superimposed on the SPM8 canonical ICBM MRI image. Color scale codes the value of the $F$ statistic at each voxel. (A, right) Parameter estimates (canonical HRF [blue] and onset latency [red] and dispersion [green] derivatives) at the peak voxel coordinate in the SMA [2, $\left.8,58 \mathrm{~mm} ; p_{\text {corr }}<.05\right]$, indicating prominent latency (delayed onset) and dispersion (increased duration) effects for NG-related BOLD responses on top of motor practice including activity for $\mathrm{G}$ items within a block. (B) Sleep-dependent modulation of NG-related brain activity. (B, left) $F$ maps $\left(p_{\text {corr }}<.05\right)$ superimposed on the SPM8 canonical ICBM MRI image. Color scale codes the value of the $F$ statistic at each voxel. (B, right) Parameter estimates of the canonical HRF (blue) with its onset (red) and dispersion (green) derivatives at peak voxels for grammatical violations-related BOLD responses during $\mathrm{S} 2$ (Day 1) as compared with S3 (Day 4) during SRT practice in the RS and SD populations. Peak coordinates for parameter estimates (blue crosshairs on the left) is located in the right cuneus $(2,-82,40, \mathrm{~mm})$, showing normalization of delayed BOLD response onset at Day 4 (S3) in the RS group, whereas onset becomes delayed at Day 4 in the SD group. Error bars are standard deviations.

\section{A NG-related brain activity across S1 and S2 SMA $\left[2,8,58 \mathrm{~mm} ; p_{\text {corr }}<.05\right]$}

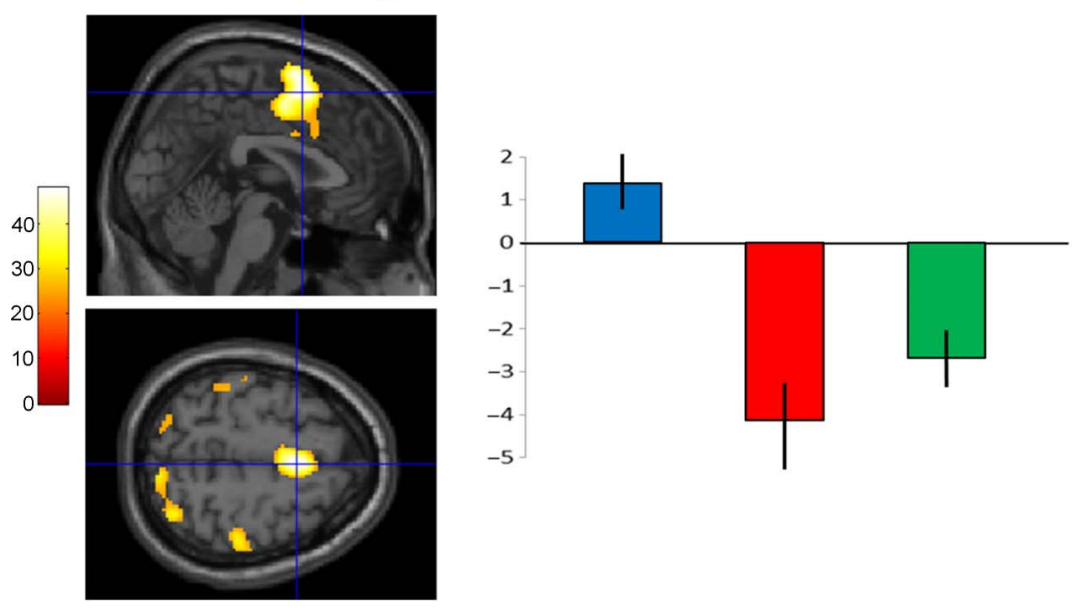

B Sleep-dependent modulation of NG-related brain activity (S2 vs. S3) Cuneus $\left[2,-82,40 \mathrm{~mm} ; p_{\text {corr }}<.05\right]$

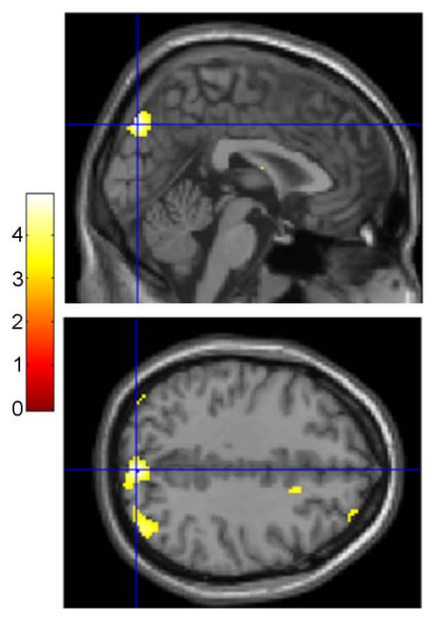

S2

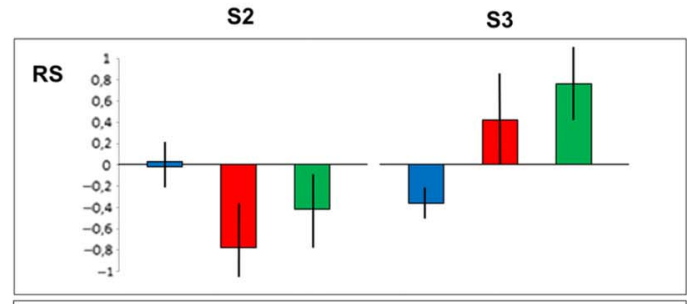

SD

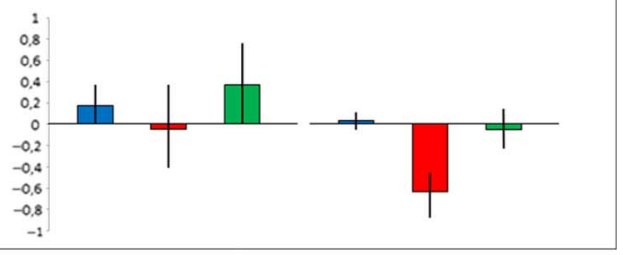

each session (S1 and S2 at Day 1, S3 at Day 4). Outliers ( \pm 2 SDs from the mean), incorrect responses, and the first five trials in each block were discarded from the analyses. A repeated-measure ANOVA was conducted on valid RTs with within-subject factors Grammaticality (G vs. NG), Session (S1 vs. S2 vs. S3), and Blocks (blocks 1-24) and between-subject factor Sleep (RS vs. SD). The analysis disclosed a main effect of Grammaticality (faster RTs for $G$ than NG stimuli; $F(1,22)=47.88)$ indicating successful learning of the sequential regularities. Session and blocks effects were also significant (gradual RT improvement from one session to the next, $F(2,44)=$ 76.66 , and across blocks, $F(23,506)=7.87$; all $p s<$
$.0001)$, indicating progressive visuo-motor skill adaptation. The main effect of Sleep was not significant $(p>$ $.88)$. There was also a Grammaticality $\times$ Session interaction effect $(F(2,44)=3.58, p=.036)$ with a marginal increase in grammatical differentiation from S1 to S3 indicative of sequence learning evolution but no Grammaticality $\times$ Session $\times$ Sleep or Session $\times$ Sleep interaction (all $p s>$.68; see Figure 2). Additional analyses restricted to the four blocks performed at the end of training (S2, Day 1) and at the beginning of testing (S3, Day 4) or conducted on RTs' variation coefficients or accuracy (percentage of correct responses) equally failed to disclose a main effect of the sleep factor or any 
interaction effect between the sleep, grammaticality, and session factors. Nevertheless, there was a trend (least significant difference post hoc test, $p=.06$ ) for RTs' variation coefficients to be smaller at Day 4 (S3) than at Day 1 (S2), like in our prior study (Peigneux et al., 2003) in which no sleep deprivation group was actually tested. Consequently, our results are essentially in agreement with previous behavioral reports (Nemeth et al., 2010; Robertson et al., 2004) having led to the conclusion that time more than sleep participates in the consolidation of implicitly learned regularities.

\section{fMRI Analyses}

\section{Rules Violation-related BOLD Responses}

A first analysis aimed at unraveling cerebral responses elicited by NG items violating sequential rules across S1 and S2 (Day 1). Results revealed modified BOLD responses on NG stimuli presentation during SRT practice in an extended cortico-cerebellar network including the SMA (peak voxel coordinate in MNI space $=2,8,58 \mathrm{~mm}$; $\left.Z=6.41 ; p_{\text {corr }}<.05\right)$ and associated motor cortices, superior parietal and occipital cortex and the cerebellum (Crus I). Additionally, we found bilateral activations in the caudate nucleus ( $-16,0,14 \mathrm{~mm}: Z=3.51 ; 14,4,6 \mathrm{~mm}$ : $Z=3.48)$ and the putamen $(-18,14,-6 \mathrm{~mm}: Z=3.44$; 12, 12, $-4 \mathrm{~mm}: Z=3.86$; all $\left.p \mathrm{~s}_{\mathrm{svc}(10 \mathrm{~mm})}<.05\right)$ around previously reported coordinates of BG structures involved in probabilistic sequence learning (Peigneux et al., 2000). Negative derivative parameter estimates showed that NG-related effects on brain activity are mostly characterized by delayed onset latency and larger temporal dispersion of the BOLD response on top of motor practice including activity for $\mathrm{G}$ items (85\%) within a block (Figure 3A).

\section{Sleep-dependent Modulation of Learning-related Brain Activity}

In a second analysis, we tested the effect of posttraining sleep on the evolution of learning-related cerebral activity. Planned comparisons tested differential changes in NGrelated BOLD responses from S2 at Day 1 to S3 at Day 4 between participants allowed to sleep (RS) and deprived of sleep (SD) on the posttraining night.

Interaction effects with the sleep factor were found in the right cuneus $\left(2,-82,40 \mathrm{~mm} ; p_{\text {corr }}<.05\right)$. In this structure, inspection of derivative parameters showed that delayed onset latency and increased dispersion of NG-related BOLD responses normalized at Day 4 in the RS group. Conversely, BOLD response latency in the SD group became more delayed and temporally extended at Day 4 than Day 1, although in both cases, the amplitude of the canonical response was not or slightly modified across groups or sessions (Figure 3B). Averaged time courses over individuals are provided for the main NGrelated coordinate (i.e., the cuneus at 2, $-82,40 \mathrm{~mm}$; $\left.p_{\text {corr }}<.05\right)$ on Figure 4 for illustrative purposes. Left shows the time course of NG-related responses (peristimulus time histogram, PSTH) over the population during learning (presleep manipulation S1 [brown] and S2 [blue] at Day 1), with a steep onset and sustained BOLD response, more developed at S2 when sequence learning is more advanced and sensitivity to NG stimuli is stronger. Right shows PSTH during postsleep manipulation S3 at Day 4 in the RS (pink) and SD (gray) conditions with delayed onset latency of NG-related BOLD responses in the SD as compared with the RS session but with fairly similar amplitude.

Similar effects were observed in cortical and subcortical areas around a priori coordinates (Table 1) taken from prior studies having shown experience-dependent continuation of learning-related activity during REM sleep

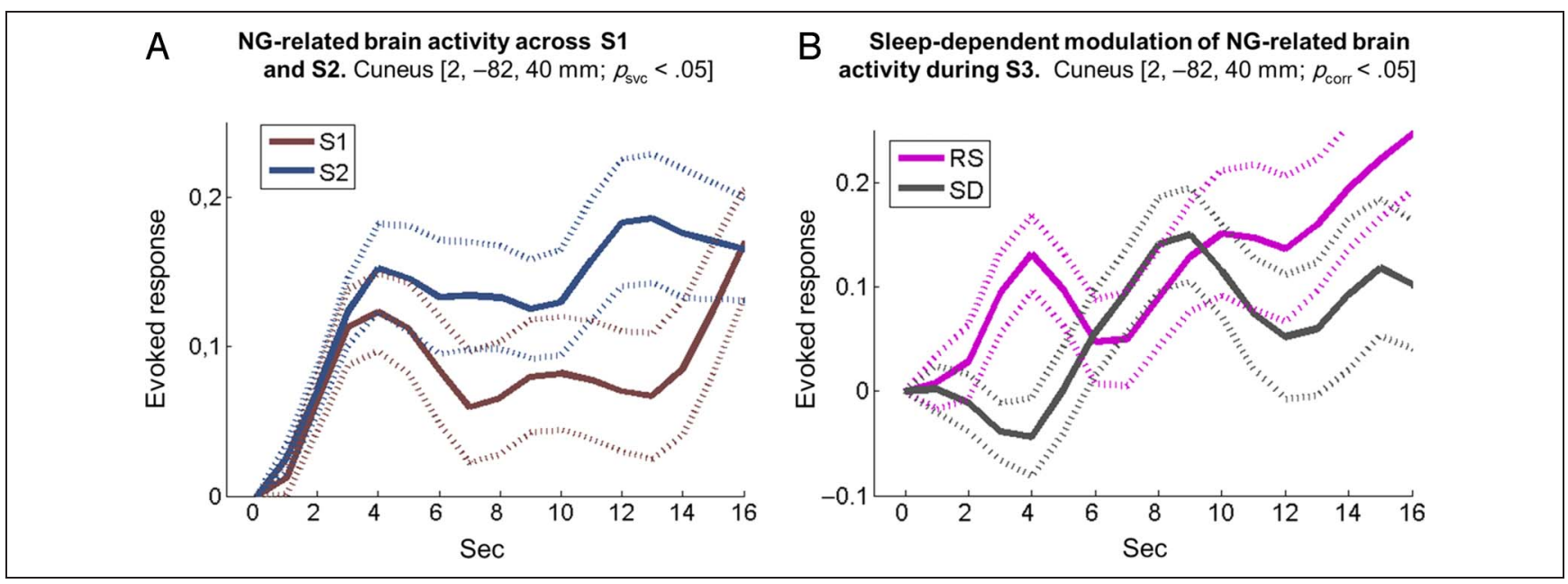

Figure 4. Averaged time courses over individuals for the main NG-related coordinate (i.e., the cuneus at 2, $-82,40 \mathrm{~mm} ; p_{\text {corr }}<.05$ ). (A) Time courses of NG-related responses (PSTH) over the population during learning (presleep manipulation S1 [brown] and S2 [blue] at Day 1). (B) Time courses of NG-related responses (PSTH) during postsleep manipulation S3 at Day 4 in the RS (pink) and SD (gray) conditions. 
Table 1. Sleep-dependent Modulation of Learning-related Brain Activity

\begin{tabular}{|c|c|c|c|c|c|c|c|}
\hline \multicolumn{6}{|c|}{ Voxel Level } & \multicolumn{2}{|r|}{ Cluster Extent } \\
\hline Region & Side & $x$ & $y$ & $z$ & $Z$ & ${ }^{K}$ E Cluster & Additional Activated Areas in the Cluster \\
\hline Cuneus** & & 2 & -82 & 40 & 4.78 & 235 & Right/left superior parietal lobule (7M, 7P) \\
\hline \multirow[t]{3}{*}{ Fusiform } & Left & -28 & -62 & -6 & 4.41 & 344 & Left area 17 \\
\hline & & & & & & & Left V4 \\
\hline & & & & & & & Left area 18 \\
\hline Posterior parietal furrow & Left & -16 & -54 & 64 & 4.18 & 242 & Left superior parietal lobule $(7 \mathrm{~A}, 5 \mathrm{~L})$, area 1,2 \\
\hline Ventrolateral pFC (inferior frontal gyrus) & Right & 44 & 12 & 18 & 4.10 & 123 & Right area 44 \\
\hline Precuneus & Right & 8 & -54 & 52 & 4.09 & 67 & Right superior parietal lobule $(5 \mathrm{M}, 5 \mathrm{~L}, 7 \mathrm{~A})$ \\
\hline Thalamus & Right & 14 & -4 & 14 & 3.94 & 197 & - \\
\hline Dorsolateral pFC (middle frontal gyrus) & Right & 36 & 6 & 46 & 3.98 & 80 & - \\
\hline Dorsolateral pFC (middle frontal gyrus) & Left & -22 & 38 & 24 & 3.98 & 80 & - \\
\hline Thalamus & Right & 10 & -30 & -8 & 3.77 & 27 & \\
\hline Dorsolateral pFC (middle frontal gyrus) & Right & 26 & 38 & 24 & 3.60 & 47 & \\
\hline Precentral gyrus & Left & -48 & -2 & 34 & 3.69 & 49 & Left area $4 \mathrm{p}, 6,4 \mathrm{a}, 3 \mathrm{a}$ \\
\hline Cerebellum (crus 1) & Left & -10 & -80 & -26 & 3.58 & 72 & - \\
\hline \multirow[t]{2}{*}{ Cerebellum } & Right & 8 & -62 & -4 & 3.34 & 53 & Right area 18 \\
\hline & & & & & & & Right V3v \\
\hline Cerebellum & Left & -34 & -62 & -30 & 3.41 & 22 & - \\
\hline Amygdala & Left & -30 & 2 & -14 & 3.50 & 64 & Left amygdala (LB, SF, CM) \\
\hline Cuneus $^{\circ 1}$ & Right & 32 & -74 & 30 & 3.51 & 10 & \\
\hline Mesencephalon $^{\circ 2}$ & Right & 8 & -30 & -10 & 3.21 & 1 & \\
\hline Thalamus $^{\circ 3}$ & Right & 10 & -30 & -8 & 3.77 & 22 & \\
\hline Caudate nucleus ${ }^{\circ 4}$ & Left & -24 & -10 & 22 & 3.80 & 41 & \\
\hline Caudate nucleus $^{05}$ & Right & 14 & -6 & 14 & 3.87 & 83 & \\
\hline
\end{tabular}

Effect of posttraining sleep on the evolution of NG-related responses: comparison of overnight changes of activity (S2 vs. S3) in RS vs. SD participants, masked exclusively by the difference between SD and RS groups at presleep session S2. $x, y$, and $z$ are standard MNI coordinates (mm). $Z=Z$ statistic value. ${ }^{\mathrm{K}} \mathrm{E}$ cluster $=$ cluster extent of the activation (in number of voxels). Reported activations are statistically significant at the voxel level, $p<$ .001 (uncorrected for multiple comparisons), unless specified otherwise. Activation marked by a "**" is statistically significant at the voxel level, $p_{\text {corr }}<.05$ after correction for multiple comparisons in the whole-brain volume. Activations marked by a "o" are statistically significant at the voxel level, $p_{\mathrm{svc}}<.05$, after correction in a small volume (radius $=10 \mathrm{~mm}$ ) drawn around the coordinates of interest taken from the literature: ${ }^{\circ 1}$ right cuneus $[26,-70,24]$ (Peigneux et al., 2003), ${ }^{\circ 2}$ right mesencephalon $[-2,-36,-18]$ (Maquet et al., 2000), ${ }^{\circ 3}$ right thalamus $[(-) 16,-34,-4]($ Peigneux et al., 2003), ${ }^{\circ 4}$ left caudate nucleus [-16, -6, 24] (Peigneux et al., 2003), and ${ }^{\circ 5}$ right caudate nucleus [18, -12, 20] (Peigneux et al., 2003). LB = laterobasal; $\mathrm{SF}=$ superficial; $\mathrm{CM}=$ centromedial.

(Peigneux et al., 2003; Maquet et al., 2000), including the anterior part of the right cuneus $(32,-74,30 \mathrm{~mm}$ : $Z=3.51)$, the right mesencephalon $(8,-30,-10 \mathrm{~mm}$ : $Z=3.21)$, the right thalamus $(10,-30,-8 \mathrm{~mm}: Z=$ $3.77)$, and the caudate nucleus bilaterally ( $-24,-10$, $22 \mathrm{~mm}: Z=3.80 ; 14,-6,-14 \mathrm{~mm}: Z=3.87$; all $\left.p_{\text {svc }}<.05\right)$. Finally, Table 1 displays a set of areas in which a similar sleep-dependent modulation was observed but in which activations did not survive correction for multiple comparisons threshold and were not included in our a priori anatomical hypotheses. These latter activations are reported for the sake of completeness but will not be discussed further.

\section{Sleep-dependent Changes in Functional Integration}

Finally, we conducted a PPI analysis to test the complementary hypothesis of a functional reorganization within learning-related cerebral networks following posttraining sleep. Results showed that, in the RS group, activity in the right cuneus (source voxel at 2, $-82,40 \mathrm{~mm}$ ), where we found a sleep-dependent modulation of learning- 


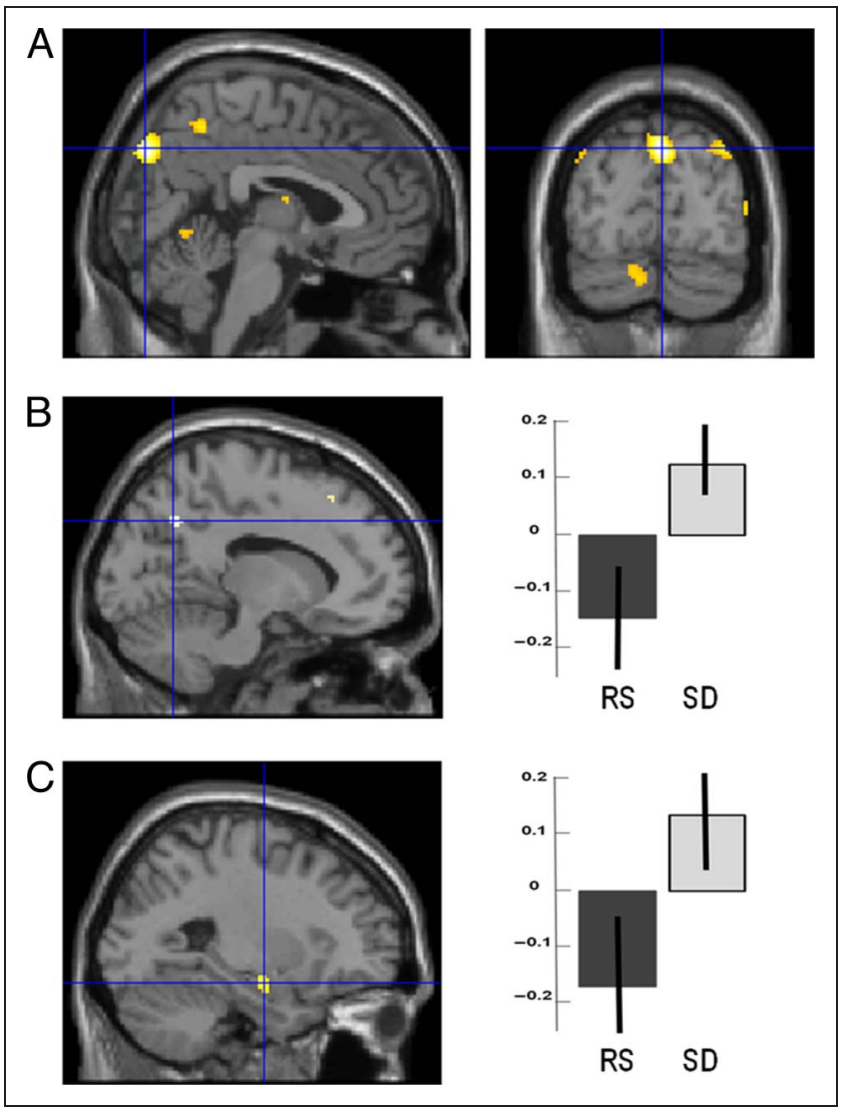

Figure 5. Sleep-dependent changes in functional integration. PPI analysis showing brain areas where activity is differentially coupled with activity in the source area of the right cuneus ([A];2, -82 , $40 \mathrm{~mm}$ in the MNI space). Interaction effects were found in the left precuneus $([\mathrm{B}] ;-12,-62,38 \mathrm{~mm})$ and the left hippocampus $([\mathrm{C}]$; $-26,-8,-16 \mathrm{~mm})$. In those areas, activity was negatively coupled with the right cuneus activity in the RS group and positively coupled in the SD group. Statistical effects are superimposed on the SPM8 canonical ICBM MRI image.

related brain activity, was negatively coupled with BOLD variations in the left precuneus $\left(-12,-62,38\right.$; $p_{\text {uncorr }}<$ $.001)$ and at a more lenient threshold in the hippocampus $\left(-26,-8,-16 ; p_{\text {uncorr }}<.002\right)$. A reverse functional pattern was found in the SD group, with a positive coupling between the right cuneus activity and the left precuneus and hippocampus (Figure 5). Of note, a similar NG-related hippocampal activation was found during the presleep learning sessions around coordinates previously associated with information tagging for future processing during sleep (i.e., 8, 18, $32 \mathrm{~mm}: Z=4.51$; 46, 28, $18 \mathrm{~mm}$ : $Z=4.98$, in Albouy et al., 2008; all $\left.p \mathrm{~s}_{\mathrm{svc}}<.0001\right)$.

\section{DISCUSSION}

In this study, we aimed at exploring the impact of posttraining sleep on the modulation and/or the reorganization of the neurophysiological processes subtending consolidation of implicit sequence learning in the probabilistic SRTT. Behavioral results showed that participants learned the underlying sequential regularities through practice, as demonstrated by slowed responses for items violating the rules (RTs: NG > G). Global RTs decreased, and grammaticality effects amplified over sessions, reflecting gradual visuo-motor skill adaptation and sequence learning, respectively, but to the same extent in participants allowed to sleep (RS) or not (SD) on the first posttraining night. Consequently, our results are in agreement with previous behavioral reports (Nemeth et al., 2010; Robertson et al., 2004) having led to the conclusion that time more than sleep participates in the consolidation of implicitly learned regularities.

Analysis of functional neuroimaging data revealed that grammatical violations in the sequence during learning sessions at Day 1 were associated with delayed onset latency and increased dispersion in BOLD responses in a large cortico-subcortical network. Previous studies showed that derivative parameters capturing limited variations in onset latency and dispersion of the canonical BOLD responses provide a valuable additional source of information about neural activity (Kellermann et al., 2011; Ford, Johnson, Whitfield, Faustman, \& Mathalon, 2005; Henson et al., 2002). For instance, Henson et al. (2002) reported that hemodynamic responses within the occipito-temporal region (associated with lexical decisions) were both smaller and slower to nonwords than to real words (as were decisions about new faces as compared with repeated faces over the fusiform gyrus), notwithstanding the fact that previous blocked designs studies revealed activations for the lexical decision task but were not able to yield evidence for differences in activation for words versus nonwords (Rumsey et al., 1997; Price et al., 1994). We surmise here that the specific modeling we applied mostly explains why our effects are not observed in terms of modulations of the amplitude of the hemodynamic canonical BOLD response, but rather, in a subtler manner by differences in time and dispersion derivatives. Indeed, studying NG stimuli on top of motor practice including activity for $\mathrm{G}$ items (85\%) within a block masked the properties commonly shared by both stimulus types, including the gross neurophysiological activity associated with motor responses, leaving visible only the differences related to the acquisition of the probabilistic rules subtending the succession of stimuli.

Results showed that the network of NG-related responses encompassed the SMA and associated motor cortices involved in response preparation and selection on the basis of external or internal information and occipito-parietal regions participating in visuospatial perception and attentional selection processes (Curran, 1995, 1998). At the subcortical level, the caudate nucleus and the putamen have been involved in the implicit automation of serial information through participation in the cortical-subcortical motor loops linking prefrontal and BG areas (Doyon et al., 2009), and cerebellar activations may subtend the monitoring of afferent sensory components and the optimization of movements using sensory 
feedback information (Doyon, Penhune, \& Ungerleider, 2003; Jueptner \& Weiller, 1998). Of note, cerebellar activations in Crus I have been also partially associated with the automation of rule-based information, although in the case of low-order rules (Balsters \& Ramnani, 2011). Moreover, learning-related activations found over striatal regions (i.e., putamen and caudate nucleus) are in agreement with prior studies that aimed at characterizing the neural correlates of implicit sequence learning using SRTTs (e.g., Destrebecqz et al., 2005; Peigneux et al., 2000).

Although the evolution of behavioral performance was not noticeably modified by the posttraining sleep condition, our results indicate a different evolution of NG-related brain responses in RS and SD participants, suggesting a sleep-dependent covert reorganization of the functional neuroanatomical patterns subtending practice in the probabilistic SRTT. At delayed testing after postlearning sleep, NG event-related BOLD responses in the cuneus did not distinguish anymore from motor practice including activity for $\mathrm{G}$ items (85\%), which was not the case for participants deprived of sleep after learning. A similar pattern was observed in a set of cortical and subcortical areas previously identified as being part of the network involved in implicit motor sequence learning and offline processing during sleep (i.e., cuneus, mesencephalon, thalamus, and caudate nucleus). For instance, increased activity in the cuneus and mesencephalon was previously reported using $\mathrm{H}_{2}{ }^{15} 0$ PET both during practice of the same probabilistic SRTT at wake and during postlearning REM sleep (Maquet et al., 2000). These previous results were in agreement with the neuronal replay hypothesis, which posits that activity in learning-related brain structures is replayed during sleep, eventually leading to the consolidation of newly acquired memories (e.g., Dave \& Margoliash, 2000; Wilson \& Mcnaughton, 1994). Additionally, cuneus and mesencephalon activity during posttraining REM sleep was found specifically related to the implicit acquisition of the probabilistic rules that defined stimulus sequences (as compared with practice on a random structure), and functional connectivity between the cuneus and BG structures also increased during postlearning REM sleep (Peigneux et al., 2003). Cuneus has been primarily associated with visual processing, although it has been also involved in various cognitive functions including cognitive control (Haldane, Cunningham, Androutsos, \& Frangou, 2008) and working memory (Bluhm et al., 2010). In particular, the cuneus participates in monitoring changes in ocular position in response to self-generated eye movements (Law, Svarer, Rostrup, \& Paulson, 1988) and is involved in the bottom-up control of visuospatial selective attention (Hahn, Ross, \& Stein, 2006), two processes that might be involved in the processing and implicit detection of grammatical violations in the sequence. Although cuneus activation was previously reported during sequential information processing at wake (e.g., Schubotz \& von Cramon, 2001), it is not commonly seen as a critical component of sequence learning, and further studies are needed to clarify its implication in probabilistic sequence processing. In this study, the topographic similitude between brain areas in which posttraining reactivations were observed during sleep (Peigneux et al., 2003; Maquet et al., 2000) and brain areas in which a sleep-dependent modulation of NG-related activity was found at Day 4 is indicative, although not conclusive, of the development of distinct neurophysiological processes in RS and SD participants, despite seemingly similar behavioral patterns. An alternative hypothesis would be that a persistent differentiation of NG-related responses at Day 4 in SD participants would reflect a disturbance in ongoing time-dependent consolidation processes because of the night of sleep deprivation, characterized by a decrease in available cognitive resources and learning abilities (see, e.g., Yoo, Hu, Gujar, Jolesz, \& Walker, 2007) and marked changes in cerebral activity (e.g., De Havas, Parimal, Soon, \& Chee, 2012).

Finally, a PPI analysis revealed that activity in the cuneus was negatively coupled with activity in the left precuneus and hippocampus after posttraining sleep, whereas a reverse pattern was found after sleep deprivation, further suggesting a posttraining sleep-dependent development of functional connectivity patterns. Although evidence for a hippocampal activation in probabilistic SRTT remains conjectural, we found a hippocampal activation during the presleep learning sessions at coordinates previously associated with information tagging for future processing during sleep (i.e., 8, 18, $32 \mathrm{~mm}: Z=4.51$; 46, 28, $18 \mathrm{~mm}$ : $Z=4.98$; all $p \mathrm{~s}_{\mathrm{svc}}<.0001$, around a priori coordinates from Albouy et al., 2008). This finding might suggest that the decreased coupling between cuneus and hippocampus in RS participants reflect a decrease in the strength of the tagging after sleep-related memory consolidation. Conversely, the reverse pattern observed in SD participants might reflect the persistence of a "to-be consolidated during sleep" tag. The precuneus, located in the posteromedial cortex of the parietal lobe, is known to support abstract cognitive processes involving visuospatial information and shifts in voluntary attention between targets (Cavanna \& Trimble, 2006), whereas the hippocampus may participate in novelty detection (Wang, Poe, \& Zochowski, 2008; Strange, Duggins, Penny, Dolan, \& Friston, 2005). In the context of the implicit acquisition of probabilistic succession rules in the sequence of stimuli, one possible explanation for distinct connectivity patterns and the fact that BOLD derivative parameters of NG-related responses were actually no more different from $G$ stimuli after sleep, but not after sleep deprivation, would be that posttraining sleep favored the processing of $G$ and NG stimuli in a more automatized manner, eventually leading to a reduction in attentional shifts when confronted with NG items violating sequential rules. This interpretation would be in line with the conclusions of an ERP study similarly showing that brain responses associated with the automatic shift of attention to unexpected stimuli in an auditory learning paradigm failed to develop after posttraining sleep deprivation as compared with sleep (Atienza, Cantero, \& 
Stickgold, 2004). These results were interpreted as suggesting a reduction in the voluntary attentional effort required for successful perceptual discrimination by facilitating the intrusion of a potentially meaningful stimulus into one's focus of attention for further evaluation.

To conclude, our results indicate that posttraining sleep impacts the neurophysiological processes that subtend the processing of implicit regularities in probabilistic sequence learning. This suggests that sleep participates in the integration of both regular and deviant stimuli in the context of the probabilistic SRTT. Further studies should investigate whether the absence of behavioral differences in sequence learning stems from a lack of sensitivity of the parameters used to assess performance and whether these differences in brain activity are preceding the development of behavioral differences that would be detected later on, at the scale of weeks or months.

\section{Acknowledgments}

This study was supported by FRSM 3.4.582.09.F and FRFC 2.4.627.09.F Grants from the Belgian Fonds National de la Recherche Scientifique (FRS-FNRS). C. U. was supported by a special grant of the Université Libre de Bruxelles (Foundation Vigneron) and ULB-ARC project "Pathophysiology of Memory Consolidation Processes." R. S., A. C., C. S., and P. M. are supported by FRS-FNRS.

Reprint requests should be sent to Charline Urbain or Philippe Peigneux, UR2NF - Neuropsychology and Functional Neuroimaging Research Unit, Université Libre de Bruxelles, Campus du Solbosch CP191 Avenue F. D. Roosevelt 50 B-1050 Bruxelles, Belgium, or via e-mail: curbain@ulb.ac.be, philippe.peigneux@ ulb.ac.be.

\section{REFERENCES}

Albouy, G., Sterpenich, V., Balteau, E., Vandewalle, G., Desseilles, M., Dang-Vu, T., et al. (2008). Both the hippocampus and striatum are involved in consolidation of motor sequence memory. Neuron, 58, 261-272.

Atienza, M., Cantero, J. L., \& Stickgold, R. (2004). Posttraining sleep enhances automaticity in perceptual discrimination. Journal of Cognitive Neuroscience, 16, 53-64.

Balsters, J. H., \& Ramnani, N. (2011). Cerebellar plasticity and the automation of first-order rules. Journal of Neuroscience, 31, 2305-2312.

Barakat, M., Doyon, J., Debas, K., Vandewalle, G., Morin, A., Poirier, G., et al. (2011). Fast and slow spindle involvement in the consolidation of a new motor sequence. Behavioural Brain Research, 217, 117-121.

Bluhm, R. L., Clark, C. R., McFarlane, A. C., Moores, K. A., Shaw, M. E., \& Lanius, R. A. (2010). Default network connectivity during a working memory task. Human Brain Mapping, 32, 1029-1035.

Buysse, D. J., Reynolds, C. F., Monk, T. H., Berman, S. R., \& Kupfer, D. J. (1989). The Pittsburgh Sleep Quality Index: A new instrument for psychiatric practice and research. Psychiatry Research, 28, 193-213.

Cajochen, C., Knoblauch, V., Wirz-Justice, A., Krauchi, K., Graw, P., \& Wallach, D. (2003). Circadian modulation of sequence learning under high and low sleep pressure conditions. Behavioural Brain Research, 151, 167-176.
Cavanna, A. E., \& Trimble, M. R. (2006). The precuneus: A review of its functional anatomy and behavioural correlates. Brain, 129, 564-583.

Cleeremans, A., \& McClelland, J. L. (1991). Learning the structure of event sequences. Journal of Experimental Psychology: General, 120, 235-253.

Cohen, D. A., Pascual-Leone, A., Daniel, Z., \& Robertson, E. M. (2005). Off-line learning of motor skill memory: A double dissociation of goal and movement. Proceedings of the National Academy of Sciences, U.S.A., 102, 18237-18241.

Cohen, D. A., \& Robertson, E. M. (2007). Motor sequence consolidation: Constrained by critical time windows or competing components. Experimental Brain Research, 177, 440-446.

Curran, T. (1995). On the neural mechanisms of sequence learning. PSYCHE: An Interdisciplinary Journal of Research on Consciousness (On-line), 2, 12.

Curran, T. (1998). Implicit sequence learning from a cognitive neuroscience perspective. What, how, and where? In M. A. Stadler \& P. A. Frensch (Eds.), Handbook of implicit learning (pp. 365-400). Newbury Park, CA: Sage.

Dave, A. S., \& Margoliash, D. (2000). Song replay during sleep and computational rules for sensorimotor vocal learning. Science, 290, 812-816.

De Havas, J. A., Parimal, S., Soon, C. S., \& Chee, M. W. (2012). Sleep deprivation reduces default mode network connectivity and anti-correlation during rest and task performance. Neuroimage, 59, 1745-1751.

Debas, K., Carrier, J., Orban, P., Barakat, M., Lungu, O., Vandewalle, G., et al. (2010). Brain plasticity related to the consolidation of motor sequence learning and motor adaptation. Proceedings of the National Academy of Sciences, U.S.A., 107, 17839-17844.

Destrebecqz, A., Peigneux, P., Laureys, S., Degueldre, C., Del Fiore, G., Aerts, J., et al. (2005). The neural correlates of implicit and explicit sequence learning: Interacting networks revealed by the process dissociation procedure. Learning \& Memory, 12, 480-490.

Doyon, J., Bellec, P., Amsel, R., Penhune, V., Monchi, O., Carrier, J., et al. (2009). Contributions of the basal ganglia and functionally related brain structures to motor learning. Behavioural Brain Research, 199, 61-75.

Doyon, J., Penhune, V., \& Ungerleider, L. G. (2003). Distinct contribution of the cortico-striatal and cortico-cerebellar systems to motor skill learning. Neuropsychologia, 41, 252-262.

Ellis, B. W., Johns, M. W., Lancaster, R., Raptopoulos, P., Angelopoulos, N., \& Priest, R. G. (1981). The St. Mary's Hospital sleep questionnaire: A study of reliability. Sleep, 4, 93-97.

Fischer, S., Drosopoulos, S., Tsen, J., \& Born, J. (2006). Implicit learning-Explicit knowing: A role for sleep in memory system interaction. Journal of Cognitive Neuroscience, 18, 311-319.

Fischer, S., Hallschmid, M., Elsner, A. L., \& Born, J. (2002). Sleep forms memory for finger skills. Proceedings of the National Academy of Sciences, U.S.A., 99, 11987-11991.

Fischer, S., Wilhelm, I., \& Born, J. (2007). Developmental differences in sleep's role for implicit off-line learning: Comparing children with adults. Journal of Cognitive Neuroscience, 19, 214-227.

Ford, J. M., Johnson, M. B., Whitfield, S. L., Faustman, W. O., \& Mathalon, D. H. (2005). Delayed hemodynamic responses in schizophrenia. Neuroimage, 26, 922-931.

Friston, K. J., Buechel, C., Fink, G. R., Morris, J., Rolls, E., \& Dolan, R. J. (1997). Psychophysiological and modulatory interactions in neuroimaging. Neuroimage, 6, 218-229. 
Friston, K. J., Fletcher, P., Josephs, O., Holmes, A., Rugg, M. D., \& Turner, R. (1998). Event-related fMRI: Characterizing differential responses. Neuroimage, 7, 30-40.

Gais, S., Albouy, G., Boly, M., Dang-Vu, T. T., Darsaud, A., Desseilles, M., et al. (2007). Sleep transforms the cerebral trace of declarative memories. Proceedings of the National Academy of Sciences, U.S.A., 104, 18778-18783.

Gläscher, J. (2009). Visualization of group inference data in functional neuroimaging. Neuroinformatics, 7, 73-82.

Hahn, B., Ross, T. J., \& Stein, E. A. (2006). Neuroanatomical dissociation between bottom-up and top-down processes of visuospatial selective attention. Neuroimage, 32, 842-853.

Haldane, M., Cunningham, G., Androutsos, C., \& Frangou, S. (2008). Structural brain correlates of response inhibition in bipolar disorder. International Journal of Psychopharmacology, 22, 138-143.

Henson, R. N. A., Price, C. J., Rugg, M. D., Turner, R., \& Friston, K. J. (2002). Detecting latency differences in event-related bold responses: Application to words versus nonwords and initial versus repeated face presentations. Neuroimage, 15, 83-97.

Horne, J. A., \& Ostberg, O. (1976). A self-assessment questionnaire to determine morningness-eveningness in human circadian rhythms. International Journal of Chronobiology, 4, 97-110.

Jimenez, L., Mendez, C., \& Cleeremans, A. (1996). Comparing direct and indirect measures of sequence learning. Journal of Experimental Psychology: Learning, Memory, and Cognition, 22, 948-969.

Jueptner, M., \& Weiller, C. (1998). A review of differences between basal ganglia and cerebellar control of movements as revealed by functional imaging studies. Brain, 121, 1437-1449.

Kellermann, T., Reske, M., Jansen, A., Satrapi, P., Shah, J., Schneider, F., \& Habel, U. (2011). Latencies in bold response during visual attention processes. Brain Research, 1386, 127-138.

Laureys, S., Peigneux, P., Phillips, C., Fuchs, S., Degueldre, C., Aerts, J., et al. (2001). Experience-dependent changes in cerebral functional connectivity during human rapid eye movement sleep. Neuroscience, 105, 521-525.

Law, I., Svarer, C., Rostrup, E., \& Paulson, O. B. (1988). Parieto-occipital cortex activation during self-generated eye movements in the dark. Brain, 121, 2189-2200.

Maquet, P., Laureys, S., Peigneux, P., Fuchs, S., Petiau, C., Phillips, C., et al. (2000). Experience-dependent changes in cerebral activation during human REM sleep. Nature Neuroscience, 3, 831-836.

McGaugh, J. L. (2000). Memory-A century of consolidation. Science, 287, 248-251.

Nemeth, D., Janacsek, K., Londe, Z., Ullman, M. T., Howard, D. V., \& Howard, J. H. (2010). Sleep has no critical role in implicit motor sequence learning in young and old adults. Experimental Brain Research, 201, 351-358.

Oldfield, R. C. (1971). The assessment and analysis of handedness: The Edinburgh inventory. Neuropsychologia, 9, 97-113.

Orban, P., Rauchs, G., Balteau, E., Degueldre, C., Luxen, A., Maquet, P., et al. (2006). Sleep after spatial learning promotes covert reorganization of brain activity. Proceedings of the National Academy of Sciences, U.S.A., 103, 7124-7129.
Peigneux, P., Laureys, S., Fuchs, S., Destrebecqz, A., Collette, F., Delbeuck, X., et al. (2003). Learned material content and acquisition level modulate cerebral reactivation during posttraining rapid-eye-movements sleep. Neuroimage, 20, 125-134.

Peigneux, P., Maquet, P., Meulemans, T., Destrebecqz, A., Laureys, S., Degueldre, C., et al. (2000). Striatum forever despite sequence learning variability: A random effect analysis of PET data. Human Brain Mapping, 10, 179-194.

Penny, W., \& Holmes, A. (2003). Random-effect analysis. In R. Frackowiak, K. Friston, C. Frith, R. Dolan, C. Price, S. Zeki, J. Ashburner, \& W. Penny (Eds.), Human brain function (2nd ed., pp. 725-760). London: Academic Press.

Price, C. J., Wise, R. J., Watson, J. D., Patterson, K., Howard, D., \& Frackowiak, R. S. (1994). Brain activity during reading. The effects of exposure duration and task. Brain, 117, 1255-1269.

Rauchs, G., Orban, P., Schmidt, C., Albouy, G., Balteau, E., Degueldre, C., et al. (2008). Sleep modulates the neural substrates of both spatial and contextual memory consolidation. PLOS ONE, 3, e2949.

Robertson, E. M. (2009). From creation to consolidation: A novel framework for memory processing. PLoS Biology, 7, e1000019.

Robertson, E. M., Pascual-Leone, A., \& Press, D. Z. (2004) Awareness modifies the skill learning benefits of sleep. Current Biology, 14, 208-212.

Rumsey, J. M., Horwitz, B., Donohue, B. C., Nace, K., Maisog, J. M., \& Andreason, P. (1997). Phonological and orthographic components of word recognition. A PET-rCBF study. Brain, 120, 739-759.

Schubotz, R. I., \& von Cramon, D. Y. (2001). Interval and ordinal properties of sequences are associated with distinct premotor areas. Cerebral Cortex, 11, 210-222.

Smith, C., \& MacNeill, C. (1994). Impaired motor memory for a pursuit rotor task following stage 2 sleep loss in college students. Journal of Sleep Research, 3, 206-213.

Song, S. (2009). Consciousness and the consolidation of motor learning. Behavioural Brain Research, 196, 180-186.

Song, S., Howard, J. H., \& Howard, D. V. (2007). Sleep does not benefit probabilistic motor sequence learning. Journal of Neuroscience, 27, 12475-12483.

Spencer, R. M., Gouw, A. M., \& Ivry, R. B. (2007). Age-related decline of sleep-dependent consolidation. Learning $\mathcal{E}$ Memory, 14, 480-484.

Spencer, R. M., Sunm, M., \& Ivry, R. B. (2006). Sleep-dependent consolidation of contextual learning. Current Biology, 16, 1001-1005.

Strange, B. A., Duggins, A., Penny, W., Dolan, R., \& Friston, K. (2005). Information theory, novelty and hippocampal responses: Unpredicted or unpredictable? Neural Networks, 18, 225-230.

Wang, J. X., Poe, G., \& Zochowski, M. (2008). From network heterogeneities to familiarity detection and hippocampal memory management. Physical Review E: Statistical, Nonlinear, and Soft Matter Physics, 78, 041905.

Wilson, M. A., \& McNaughton, B. L. (1994). Reactivation of hippocampal ensemble memories during sleep. Science, 265, 676-679

Yoo, S. S., Hu, P. T., Gujar, N., Jolesz, F. A., \& Walker, M. P. (2007). A deficit in the ability to form new human memories without sleep. Nature Neuroscience, 10, 385-392. 